\title{
Magma Ascent by Porous Flow
}

\author{
DAVID R. SCOTT \\ Seismological Laboratory, California Institute of Technology, Pasadena
}

David J. Stevenson

Division of Geological and Planetary Sciences, California Instutute of Technology, Pasadena

\begin{abstract}
Porous flow of buoyant liquid through partially molten rock is regarded as the initial transport process leading to magma segregation in the mantle. Recent work has identified the importance of matrix deformation and compaction in this process. We present finite-difference calculations based on a generalized form of Darcy's law that includes matrix deformation, in two-dimensional Cartesian and cylindrical coordinates. We emphasize the existence of solitary wave solutions, called magmons. These waves are regions of locally high porosity that ascend through regions of low, uniform porosity. They differ from diapirs, where the liquid and matrix ascend together. The one-dimensional waves that were previously reported are found to be unstable in two dimensions, breaking down to form two-dimensional waves of circular cross section. Both the development of the instability and the form of the two-dimensional waves are supported by theoretical analysis. Numerical experiments in which two-dimensional waves collide show that the extent to which they are conserved after collision depends on the lateral offset of the waves. The conservation can be very good; in other cases, larger waves appear to grow at the expense of smaller waves. Although the quantification of the relevant permeability and rheological parameters remains uncertain, geophysically plausible estimates suggest wavelengths of kilometers and velocities of centimeters per year. In our preliminary assessment of the relevance of these results to igneous processes, we find that magmons are unlikely to be important in regions of broad upwelling, such as beneath spreading centers. Here the transport of liquid adjusts to match the supply by melting, and compaction processes are not important. If liquid is supplied from below to a stable partially molten region of the asthenosphere, it is expected to ascend in magmons. This may occur beneath oceanic volcanic centers or in subcontinental mantle. It is possible that magma ascent in magmons leads to spatial and temporal episodicity of volcanic activity, if higher level processes do not obscure this influence. The waveform of a magmon ascends faster than the liquid within the magmon, so new liquid is taken in from above, while original liquid is lost from below. Consequently, magmons can mobilize small degrees of partial melt and deliver it rapidly to the surface.
\end{abstract}

\section{INTRODUCTION}

The initial process in the genesis of igneous rock is the partial melting of some source rock in the earth. The magma must then ascend and segregate from its parent rock by processes that result in emplacement of the magma at or near the earth's surface. Experimental observations of candidate mantle rocks [Waff and Bulau, 1979; Vaughan et al., 1982] show that the liquid can form an interconnected network within the crystalline matrix; the liquid could therefore flow relative to the matrix. This has motivated us to regard buoyancy-driven porous flow as the initial transport process leading to melt segregation.

Other transport processes will doubtless take over at later times. For example, transport through the brittle lithosphere probably occurs through cracks [Weertman, 1971; Shaw, 1980; Stevenson, 1983; Spera, 1984; Spence and Turcotte, 1985]. The porous flow process is particularly interesting because the melt is in intimate contact with the matrix through which it is flowing. The geochemical signature acquired by the melt during porous flow may be observable; in particular, it may be distinct from the signatures of higher level processes involving larger conduits or

Copyright 1986 by the American Geophysical Union.

Paper number 5B5583.

0148-0227/86/005B-5583\$05.00 magma chambers, where fractional crystallization will dominate.

In considering the porous flow process, it is important to note that melting of the parent rock may be occurring at the same time. In different settings, either the melting rate or the transport process may be the rate-limiting factor in magma supply. In the former case, the compaction process is unimportant and Darcy's law is an adequate description of the porous flow. In the latter case, Darcy's law must be modified to include the effect of matrix deformation. Recently, studies of this system [Scott and Stevenson, 1984; Richter and McKenzie, 1984] have led to the identification of solitary wave solutions, consisting of localized regions of high porosity that ascend through the porous matrix. In this paper we extend our study of these solutions to two spatial dimensions and make a preliminary assessment of their impact on igneous processes.

\section{Physical Description of the Compaction Process}

The missing element in a physical model of the buoyant ascent of a liquid by porous flow has been a description of the deformation and compaction of the matrix. This is necessary because the liquid cannot escape from a closed porous system unless the matrix compacts to fill the space it leaves. This description has been supplied by several authors recently [Scott and Stevenson, 1984; McKenzie, 1984; Fowler, 1984]. In particular, a definitive introduction 
has been provided by McKenzie [1984]. Our preferred formulation differs in its general approach from that of McKenzie, although the resulting set of governing equations are essentially the same. We reserve the details of this formulation for Appendix A. The equations that govern the coupled flow of the liquid and the deforming matrix, taken from Appendix A, are

Equation (A2)

$$
\frac{\partial f}{\partial t}=\frac{\partial}{\partial x_{i}}\left[(1-f) V_{i}\right]
$$

Equation (A 15)

$$
\begin{aligned}
V_{\imath}=\frac{k}{\eta^{l}}\left[-g \Delta \rho \delta_{i z}\right. & +\frac{\partial}{\partial x_{j}}\left(\sigma_{\imath \jmath}+p^{l} \delta_{\imath \jmath}\right) \\
& \left.-\frac{1}{(1-f)} \frac{\partial f}{\partial x_{J}}\left(\sigma_{l \jmath}+p^{l} \delta_{l j}\right)\right]
\end{aligned}
$$

Equation (A2) is the continuity condition for the two-phase system, and equation (A15) is a modified form of Darcy's law. To close this system, we must provide a description of the matrix rheology and the matrix permeability. These are discussed below.

\subsection{Rheological Description of the Matrix}

The novel feature of this physical model is the dynamic deformation of the matrix. It is therefore natural to start by considering the rheological description of the matrix. Take an isotropic and locally uniform element of the matrix, large compared to the grain size but small compared to the scale length of variations in quantities such as the porosity and grain size. The rheological behavior of this element can be divided into two types of flow.

The first is simple shear, with no compaction occurring, described by the constitutive relation:

$$
\sigma_{i j}^{\prime}=\eta\left(\cap \dot{e}_{i j}\right.
$$

The shear stress is related to the shear strain rate by a viscosity $\eta$, which will be a function of porosity. Specifically, $\eta$ is expected to decrease as the porosity increases. This decrease is partly due to the reduction of material in the matrix framework and partly due to liquidenhanced creep processes.

The second type of flow is pure compaction. The porosity is reduced, and liquid is expelled due to a different state of stress in the matrix and the liquid. Unlike McKenzie, we explicitly relate the compaction to a difference between the pressure in the solid matrix, $p^{s}$, and that in the liquid, $p^{I}$, since we believe this clarifies the physics of the process:

$$
p^{l}-p^{s}=\zeta(f) \dot{e}_{k k}
$$

This defines a bulk viscosity $\zeta$, relating the rate of compaction to the pressure difference between the two phases. We note that it is different from the bulk viscosity of a single compressible phase, although it is mathematically similar. The flow associated with this viscosity is occurring on the scale of the grain size. This microscopic flow could involve whole grain deformation, grain boundary diffusion, and liquid-enhanced processes around the pore space.

The constitutive forms above can be combined in a single constitutive relation:

$$
\sigma_{i j}+p^{l} \delta_{i j}=\sigma_{i j}^{\prime}+\left(p^{l}-p^{s}\right) \delta_{i j}=(\zeta-2 / 3 \eta) \dot{e}_{k k} \delta_{i j}+2 \eta \dot{e}_{i j}
$$

The constitutive relation is like that used for a compressible fluid, except that the liquid pressure appears as a reference for the pressure in the solid matrix. Compaction should not occur when these are equal.

In Appendix B, we analyze a simple dynamic model of the microscopic compaction of the matrix. This is presented to clarify the physical meaning of $\zeta$ and $\eta$ and to provide an understanding of the important role of the pressure difference between the matrix and the liquid. A realistic model of the compaction process would be much more complex, but this simple model illustrates three features that realistic models should share:

1. The pressure difference between the solid and liquid drives the compaction. This is true even if the compaction mechanism involves grain boundary transport or pressure solution.

2. Terms of order $f$ relative to the main terms (such as the third term on the right-hand side of (A15) above) may exist but cannot be computed with confidence. Different assumptions are found to lead to different terms of this order. Thus different theoretical approaches are only likely to agree in the limit $f \rightarrow 0$.

3. The value of $\zeta$ and $\eta$ may vary strongly with the porosity $f$, when $f \ll<1$. For example, the viscosities may vary as $f^{-m}$, with exponent $m$ lying betwen 0 and 1 .

We know of two experimental studies that provide estimates of the rheological parameters $\eta$ and $\zeta$. The first is that of Cooper and Kohlstedt [1984]. The purpose of these experiments was to investigate the importance of liquidenhanced processes in the compaction of an olivine aggregate; the liquid used was a synthetic silicate of basaltic composition. The compaction process differed from that defining $\zeta$ because it occurred through the collapse of void space, not through the expulsion of liquid. However, the results are important because they demonstrate that the rate-limiting process in the compaction is transport along solid-solid grain boundaries, where the liquid is absent. There is a modest increase in the rate of compaction when liquid is present because the diffusion path in the grain boundaries is shortened by the presence of liquid in the pores. The grain size used in these experiments was around $10 \mu \mathrm{m}$, so we cannot directly obtain a value of the bulk viscosity applicable to coarse-grained mantle rocks. The second study is that of Auer et al. [1981], in which they subjected partially molten fine-grained granite to moderate deviatoric stresses, under conditions of much greater hydrostatic stress. They also concluded that the creep rate was limited by solid-state processes, up to a critical value of around $20 \%$ liquid. At this point the shear viscosity drops due to loss of structural integrity of the matrix, as proposed by $A r z i$ [1978]. In light of these studies, we propose values for $\eta$ and $\zeta$ equal to the likely shear viscosity of crystalline mantle material close to the solidus, perhaps $10^{19} \mathrm{~Pa} \mathrm{~s}$, reflecting a small degree of liquid-enhancement.

\subsection{Permeability of the Matrix}

A second material property, the permeability of a partially molten rock, is also unknown. The functional dependence of the permeability $k$ on the porosity $f$ has a major impact on quantitative aspects of porous flow phenomena. To condense various arguments, consider a simple form for the permeability: 

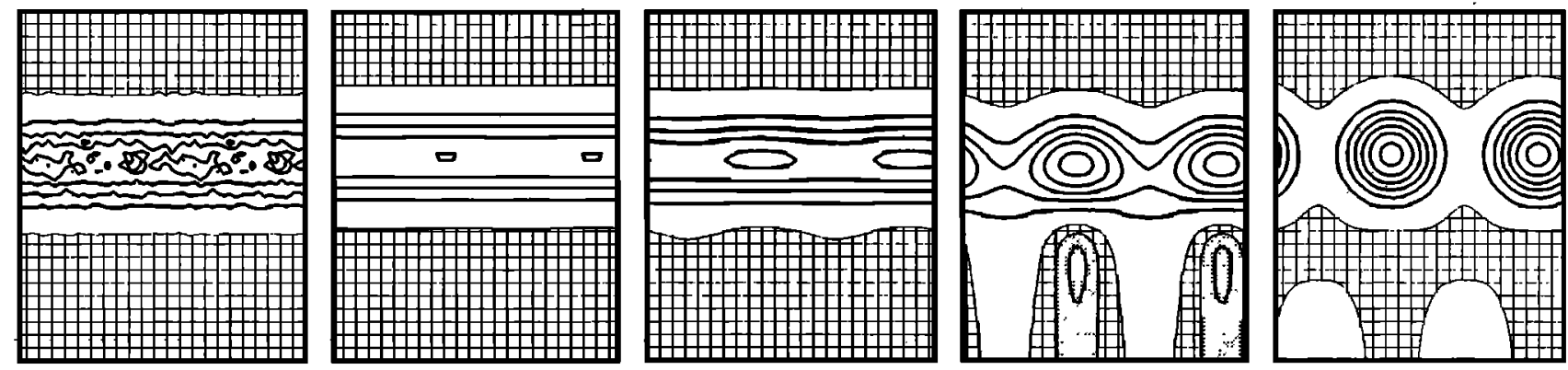

Fig. 1. The development of two-dimensional solitary waves. Each frame is a contour plot of liquid fraction in twodimensional space. The contouring scheme is as follows: the checked pattern shows values within $2 \%$ of $f_{0}=0.01$; the white area shows higher values, contoured with an interval of $0.2 f_{0}$; values less than $98 \%$ of $f_{0}$ are stippled, and contoured with an interval of $0.02 f_{0}$, one-tenth of that in the white areas. This scheme is designed to illustrate the gross behavior of the system and any small fluctuations about $f_{0}$ This experiment is performed on a two-dimensional Cartesian grid using equations (A16a) and (A16b); the numerical method is described in Appendix C. The lateral boundary conditions are periodic; each frame shows two periods for clarity. The grid scrolls upward, ascending 1.56 times the frame height between each frame. The initial state, shown in the first frame, is a one-dimensional solitary wave with white noise added to the waveform (but not to the background value $f_{0}$ ). This distribution is unstable in two dimensions; the liquid localizes laterally as the waveform ascends. The width of the grid was chosen after performing an experiment on a wider grid to establish the preferred lateral wavelength of the instability. This wavelength and the growth rate of the instability are consistent with the linear stability results shown in Figure 4 . The nondimensional height of each frame is 0.06 . The nondimensional ascent velocity of the frame is $4.7 \times 10^{-4}$. The total elapsed time is 800.

$$
k=\frac{a^{2} f^{n}}{b}
$$

where $a$ is the grain size and $n$ and $b$ are dimensionless parameters. The notation is that used by Richter and McKenzie [1984].

We feel that there is theoretical and experimental justification for a value of $n$ between 2 and 3 for partially molten rocks of low $(<20 \%)$ porosity. The Blake-KozenyCarman equation is widely used to model the permeabilities of porous systems. The theoretical basis of this equation, described by Carman [1937], dictates that the exponent $n$ is determined by the functional dependence of the mean hydraulic radius on porosity. The mean hydraulic radius is the reciprocal of the surface area of the pore space per unit volume; it is therefore an inverse measure of the resistance of the network to flow. This assumption leads to $n=2$ for a network of randomly oriented tubes of constant cross section and to $n=3$ for a bed of packed spheres. (Strictly, $k$ varies as $f^{3} /(1-f)^{2}$ in this case; the denominator has negligible effect when $f<<1$.) The texture of partially molten ultramafic rocks reported by Waff and Bulau [1979] and Vaughan et al. [1982] lies, subjectively, between these two cases. The experiments of Maalce and Scheie [1982] support this qualitative argument. They determined the rate of flow of liquids through a model porous system of compacted glass beads, intended to imitate the texture of partially molten rock. For porosities in the range between $0.5 \%$ and $20 \%$, the data are fit best by a value of $n$ between 2 and 3.

The value of the multiplicative constant $b$ is less critical. Its value is partly determined by the tortuousity of the network, expressed by the ratio of the distance between two points in the system and the actual distance traveled by the liquid between those points.

As noted by McKenzie [1984], it is likely that the porous network remains interconnected down to very small porosities, at least for the olivine-basalt systems that have been studied experimentally. This is due to the control of surface energies on the microscopic texture of the partial melt
Toramaru and Fujii [this issue] have suggested that the presence of orthopyroxenes in the mineral assemblage leads to nonconnectivity of the porous network. This may call for some adaptation of the permeability function used in modeling.

We should also address the problem raised by Waff [1980] arising from the control of surface tension on the microscopic texture. In his model, the macroscopic pressure differences due to buoyancy, which we balance with the viscous stresses due to flow, are everywhere balanced by microscopic pressure differences due to surface tension. Consequently, no flow occurs. This is a purely static model and may be unstable when the dynamics of flow are introduced. We cannot state this with confidence at the present due to the difficulties of linking the microscopic and macroscopic scales of the problem.

\section{NuMERICAL EXPERIMENTS}

Our main interest has been in studying the behavior and stability of the solitary wave solutions reported for the onedimensional case by Scott and Stevenson [1984]. The onedimensional solitary waves consist of regions of locally high liquid content that ascend over a constant, lower background porosity $f_{0}$. In the upper half of the wave, the matrix dilates in response to the arrival of more liquid than can be accommodated by Darcy flow. Symmetrically, the matrix compacts in the lower half. The wave is therefore accompanied by a dipole of pressure difference between the liquid and matrix. The passage of the wave leaves no disturbance in the amplitude of the background porosity $f_{0}$. Furthermore, the solitary waves behave, to a very good approximation, as solitons; this means that the individual waves are conserved in collisions between waves. Such collisions arise because the ascent velocity of the waves increases with the amplitude of the wave.

The numerical study of the governing equations has now been extended to two spatial dimensions, for both Cartesian and cylindrical coordinates. Some notes on the method are presented in Appendix C. Recalling the considerations of matrix viscosity and permeability discussed above and in 
$1.0(0.16)$

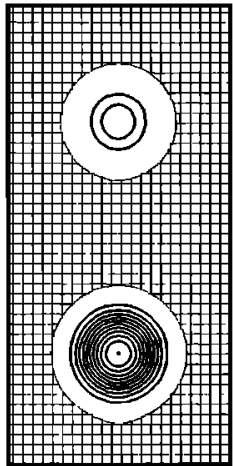

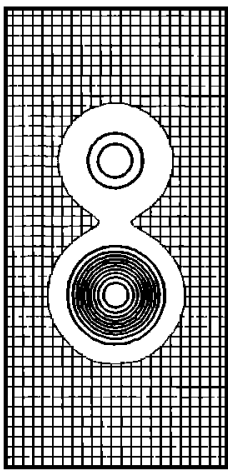

1.003

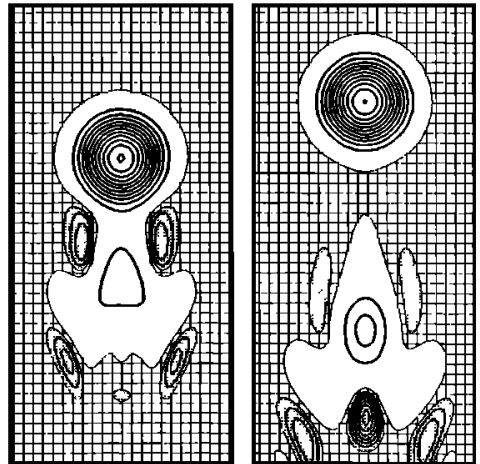

Fig. 2. A collision of cylindrically symmetric solitary waves. This sequence shows an experiment in cylindrical polar coordinates with no azimuthal variation, simulating an axially symmetric three-dimensional system. See Figure 1 for the contouring scheme; the only change is to the contour interval in the white areas, which is $0.25 f_{0}$. The experiment was performed in half the region shown in the plot, with symmetry boundary conditions across $r=0$. Equations $(\mathrm{A} 17 a)$ and $(\mathrm{A} 17 b)$ were used. The initial state consists of two spherical solitary waves, which when taken individually ascend unchanged through a region of uniform liquid fraction. The numbers above the first frame are the volumes of liquid in the large and small waves, scaled to the volume of the large wave. The number above the last frame is the volume of the large wave after the collision. The experiment shows that the collision conserves the shape and volume of the large wave very well. The smaller wave is disturbed but retains its identity. The uniform background is also somewhat disturbed. The axial symmetry means that this collision is necessarily perfectly centered. The frame ascends 0.45 times the frame height between each frame. The nondimensional height of each frame is 0.12 . The nondimensional ascent velocity of the frame is $4.5 \times 10^{-4}$. The total elapsed time is 480 .

Appendix B, the functional dependence of these quantities on $f$ must be prescribed. Studies of the one-dimensional system have showed that varying these dependences altered the results quantitatively. Qualitative aspects, notably the existence of solitary waves, were unaltered. We have therefore chosen to concentrate on a specific case for the twodimensional experiments:

$$
n=3 \quad \zeta=\eta=\text { const }
$$

This case proves to be the most analytically tractable within the physically reasonable range of choices. The constant viscosities correspond to the choice $m=0$ in our earlier report.

Less extensive experimentation on the two-dimensional system has been performed with $\zeta$ varying as $1 / f$ and $\eta=0$. In this case we assume that the strong dependence of the bulk viscosity on $f$ allows us to neglect the effects of the shear viscosity. This corresponds, in some sense, to the choice $m=1$ in our earlier report. The general features of these experiments are the same as those shown here.

The numerical experiments shown in Figures 1,2 and 3 were performed to address two specific issues that arose from the identification of solitary wave solutions in one dimension.

1. What is the fate of one-dimensional solitary waves in two dimensions? Refering to Figure 1, the one-dimensional waves are unstable in two dimensions, breaking down in the time it takes to ascend several of times their own height. Some analysis of this instability is presented in section 4.

2. Do solitary waves exist in two dimensions? Stable solitary waves of approximately circular cross-section exist in two, and probably three, spatial dimensions. Their development from a one-dimensional solution is seen in Figure 1. The waves used in the initial states in Figures 2 and 3 are all solitary waves, preserving their shape and amplitude during ascent. The circular form of the waves is supported by the analysis presented in section 5 .
3. Do two-dimensional solitary waves share the approximate soliton behavior of the one-dimensional waves when they collide? The collisions shown in Figures 2 and $3 a-3 c$ show that they retain some of their identity. In particular, a large and fast moving wave survives collisions with smaller waves quite well. It may be that large waves tend to pick up liquid from smaller waves, but this hypothesis requires further testing.

At this point we can discuss and justify the assumption (introduced at the end of Appendix A) concerning the use of local governing equations in a global frame of reference. In any of the experiments described above, we could remove the permeability of the matrix $(k=0)$ while leaving an inhomogeneous distribution of buoyancy arising from the distribution of porosity. Gravity would still drive motions in the resulting system: for example, a circular solitary wave would resemble an ascending diapir, and a Rayleigh-Taylor instability would occur in the layer in Figure 1. Such motions are excluded by the equations we have used. For the experiments presented here we can show a posteriori that these motions would be very small, with velocities less than $1 \%$ of the porous flow velocities. This may result in gradual dispersion of the solitary waves. An advantage of this approximation is that the equations are reasonably tractable to analysis, as demonstrated below.

\section{Linear Stabiltry Analysis}

In order to understand the instability shown in Figure 1, we have studied linearized forms of the governing equations. Complete linearization of the two-dimensional equations provides no interesting behavior or instability. However, it is possible to analyze infinitesimal lateral perturbations to a finite amplitude one-dimensional wave. With this analysis we identify growing instabilities whose character is consistent with the numerical finite amplitude instabilities reported above.

We proceed by writing the nondimensionalized forms of the two-dimensional Cartesian equations (A2), (A16a), and 
(a)
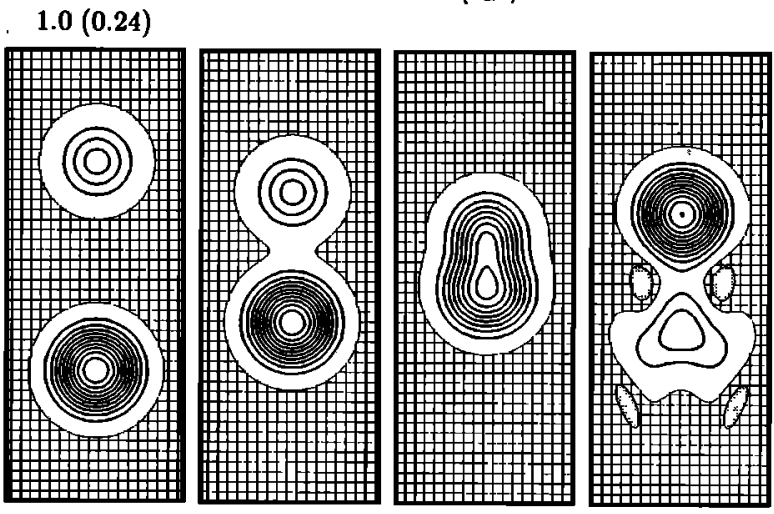

1.000

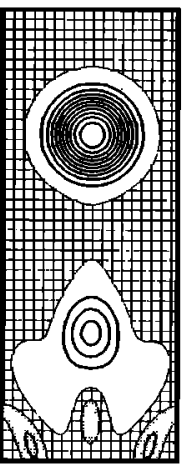

(b)

$1.0(0.24)$
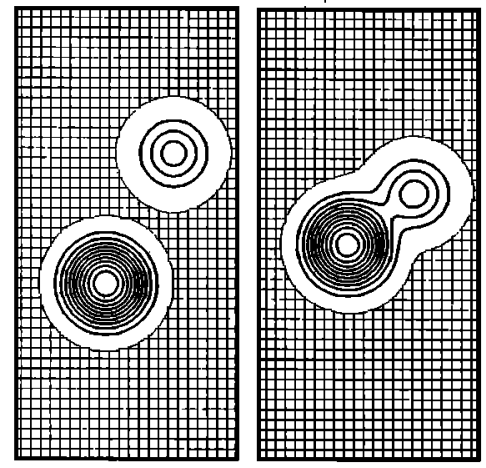

1.03
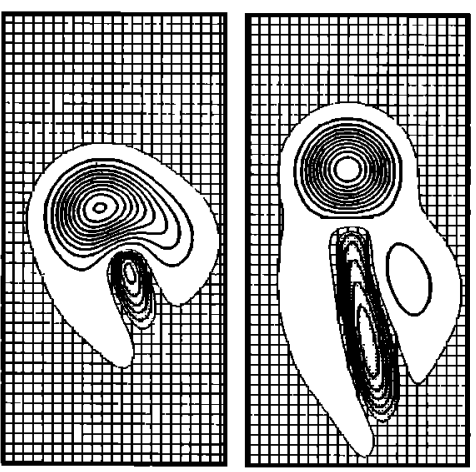

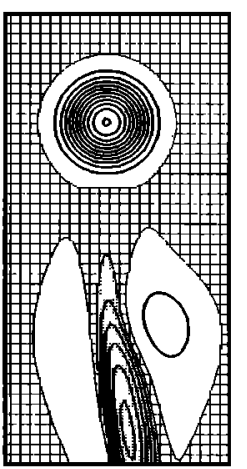

$1.0(0.24)$

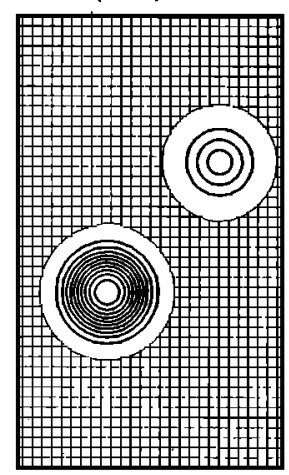

(c)

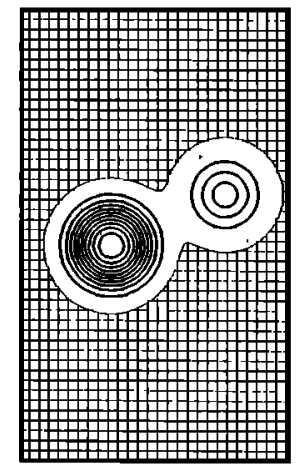

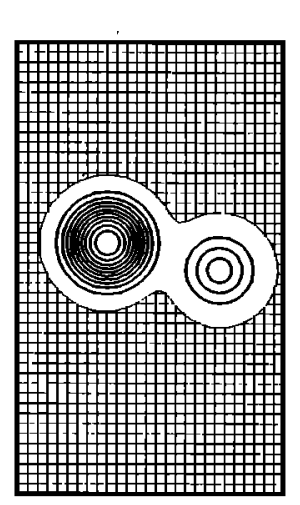

1.000

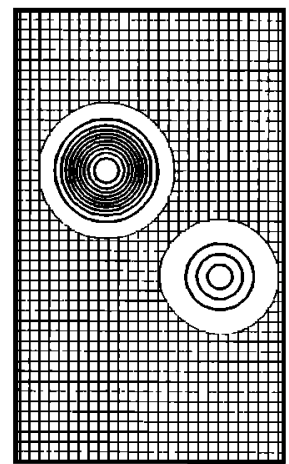

Fig. 3. Centered and offset collisions in two dimensions. The contouring scheme is the same as that in Figure 1. These experiments are in two-dimensional Cartesian coordinates with periodic boundary conditions, and use equations $(A 16 a)$ and $(A 16 b)$. The three sequences show the same pair of solitary waves, one large and one small, colliding when centered (Figure $3 a$ ) and with two offsets (Figures $3 b$ and $3 c$ ). The centered collision shown in Figure $3 a$ resembles the pseudo-three-dimensional collision in Figure 2 quite closely, which lends us some confidence in the relevance of two-dimensional experiments. As in Figure 2, note the good conservation of the volume of the larger wave. In Figure $3 c$ the lateral offset between the two waves is large enough for them to pass without interacting significantly. There is, however, a significant difference between the end results in Figures $3 a$ and $3 c$. In Figure $3 a$ the liquid within the small wave is absorbed into the large wave; the large wave loses an equivalent volume from its base, which reforms as a new small wave. In Figure $3 c$, there is no such exchange. The intermediate case is shown in Figure $3 b$. The large wave is shifted laterally during the collision; it also emerges with $3 \%$ more liquid (about $8 \%$ of the smaller wave). The small wave is severely distorted, and a second small wave is left in the wake of the large wave. They are separated by a strip depleted to about $90 \%$ of $f_{0}$. The remnant of the small wave contains its original liquid (as in Figure $3 c$ ) and the new small wave contains liquid lost from the large wave (as in Figure $3 a$ ). The frame ascends 0.41 times the frame height between each frame. The nondimensional height of each frame is 0.12 . The nondimensional ascent velocity of the frame is $4.5 \times 10^{-4}$. The total elapsed time is 480 for Figures $4 a$ and $4 b$, and 248 for Figure $4 c$. 
(A16b), with $O(f)$ terms omitted $(f<<1)$. This approximation amounts to replacing factors of $(1-f)$ by unity wherever they appear.

$$
\begin{gathered}
f_{t}=w_{z}+u_{x} \\
w=f^{n}\left[-1+w_{z z}+\alpha w_{x x}+(1-\alpha) u_{x z}\right] \\
u=f^{n}\left[u_{x x}+\alpha u_{z z}+(1-\alpha) w_{z x}\right]
\end{gathered}
$$

where $\alpha \equiv \eta /(\zeta+4 / 3 \eta)$ and subscripts denote partial differentiation. In the absence of $\mathrm{x}$ dependence, these reduce to the constant viscosity $(m=0)$ equations used in our earlier report.

The starting solution which we perturb is that for a onedimensional solitary wave, described by Scott and Stevenson [1984]. The solution has $f=\varphi(y)$ and $w=w_{0}(y)$, where $y \equiv z-c t$ and $c$ is the phase velocity of the wave. The functions $\varphi$ and $w_{0}$ therefore satisfy $-c \varphi^{\prime}=w_{0}^{\prime}$ and $w_{0}=\varphi^{n}\left[-1+w_{0}^{\prime \prime}\right]$, where a prime indicates differentiation with respect to $y$. The porosity profile $\varphi(y)$ assumes a constant, low value $\varphi_{0}$ in the background region away from the wave and has a maximum amplitude $\varphi_{\max }$. The profile is vertically symmetric about the peak. The velocity profile $w_{0}$ is related to $\varphi$ by $w_{0}=-\left[\varphi_{0}^{n}+c\left(\varphi-\varphi_{0}\right)\right]$.

In the numerical modeling we used $n=3$ (see equation (5)). For this particular case, the governing equations may be integrated twice [Richter and McKenzie, 1984] to give an implicit expression for the wave profile:

$\frac{y}{2 \sqrt{2 c \varphi_{\max }}}=\frac{\sqrt{(1-\psi / \Psi)}}{2}+\frac{1}{4 \sqrt{\Psi(\Psi-1)}} \ln \left|\frac{\sqrt{\Psi-1}+\sqrt{\Psi-\Psi}}{\sqrt{\Psi-1}-\sqrt{\Psi-\psi}}\right|(8)$

where $\psi(y) \equiv \varphi(y) / \varphi_{0}$ and $\Psi \equiv \varphi_{\max } / \varphi_{0}$. The phase velocity is given by $c=\varphi_{0}^{2}(2 \Psi+1)$, and we define the wavelength as $\lambda=2 \sqrt{2 c \varphi_{\max }}$.

Our perturbed solution to (6), (7a), and (7b) consists of the one-dimensional wave with harmonic lateral perturbations:

$$
\begin{gathered}
f=\varphi(y)+\beta(y) \sin k x e^{\sigma t} \\
w=w_{0}(y)+c \gamma(y) \sin k x e^{\sigma t} \\
u=c \delta(y) \cos k x e^{\sigma t}
\end{gathered}
$$

The functions $\beta, \gamma$, and $\delta$ are unknown, as is the growth rate $\sigma$. The objective of this analysis is to find the dependence of $\sigma$ on the lateral wavenumber $k$. Substitution of (9) into (6), (7a), and $(7 b)$ and retaining only linear terms in the small quantities $\beta, \gamma$, and $\delta$, yields

$$
\begin{gathered}
\sigma \beta-c \beta^{\prime}=c \gamma^{\prime}-k c \delta \\
\gamma=\frac{n \varphi^{n-1} \beta}{c}\left[-1+w_{0}^{\prime \prime}\right]+\varphi^{n}\left[\gamma^{\prime \prime}-\alpha k^{2} \gamma-(1-\alpha) k \delta^{\prime}\right] \\
\delta=\varphi^{n}\left[-k^{2} \delta+\alpha \delta^{\prime \prime}+(1-\alpha) k \gamma^{\prime}\right]
\end{gathered}
$$

where a prime again denotes differentiation with respect to $y$.

It is important to rescale these equations by setting the unit of length equal to the vertical wavelength of the onedimensional wave $\lambda$. The unit of time is set to $\lambda / c$. At this stage we must set $n=3$, so that we may use substitutions from the expressions for the one-dimensional wave. The equations become

$$
\begin{gathered}
\sigma \cdot \beta-\beta^{\prime}=\gamma^{\prime}-k \cdot \delta \\
8 \Psi(2 \Psi+1) \gamma=-\frac{24 \Psi}{\psi}[\Psi(2 \Psi+1)-2 \Psi] \beta \\
+\Psi^{3}\left[\gamma^{\prime \prime}-\alpha k^{2} \gamma-(1-\alpha) k_{*} \delta^{\prime}\right] \\
8 \Psi(2 \Psi+1) \delta=\psi^{3}\left[-k^{2} \delta+\alpha \delta^{\prime \prime}+(1-\alpha) k_{*} \gamma^{\prime}\right]
\end{gathered}
$$

where a prime now denotes differentiation with respect to $y_{*} \equiv y / \lambda$ and $k_{*}$ and $\sigma_{*}$ are the rescaled wave number and growth rate. The most important feature of these rescaled equations is that there is no explicit dependence on $\varphi_{0}$; the solutions are characterized solely by $\Psi$, the wave amplitude relative to the backgound porosity.

Away from the wave, as $y_{*} \rightarrow \pm \infty$ and $\psi \rightarrow 1$, the coefficients multiplying $\beta, \gamma$, and $\delta$ in these perturbation equations become constant. The asymptotic solutions must then have the form

$$
\begin{aligned}
& \beta^{ \pm}=\sum_{i=0}^{5} b_{i}^{ \pm} \exp \left(q_{i} y_{*}\right) \\
& \gamma^{ \pm}=\sum_{i=0}^{5} c_{i}^{ \pm} \exp \left(q_{i} y_{*}\right) \\
& \delta^{ \pm}=\sum_{i=0}^{5} d_{i}^{ \pm} \exp \left(q_{i} y_{*}\right)
\end{aligned}
$$

The superscripts + and - refer to $y_{*} \rightarrow \pm \infty$. Substituting these forms into (12), (13a), and (13b) gives, for each $i$, a set of three homogeneous linear equations in $b_{i}^{ \pm}, c_{l}^{ \pm}$, and $d_{l}^{ \pm}$. For a solution to exist, the determinant of the coefficients in these equations must be zero; this provides a quintic polynomial whose roots are the exponents $q_{i}$. When evaluated numerically, these roots are all found to be real. Two of the roots, designated $q_{1}$ and $q_{2}$, are always negative; two $\left(q_{4}\right.$ and $\left.q_{5}\right)$ are always positive; the remaining root $\left(q_{3}\right)$ has the same sign as $\sigma$.

Acceptable solutions for $\beta, \gamma$, and $\delta$ in equations (12), $(13 a)$, and $(13 b)$ must converge as $y_{\bullet} \rightarrow \pm \infty$. This is achieved for $\sigma>0$ if $b_{1}^{-}=b_{2}^{-}=b_{3}^{+}=b_{4}^{+}=b_{5}^{+}=0$ (and similarly for the coefficients $c_{i}^{ \pm}$and $d_{i}^{*}$. The solutions were obtained numerically, using a shooting technique. We start with a decaying solution at large positive $y_{*}$, i.e., $b_{i}=c_{i}{ }^{\ddagger} d_{i} \ddagger 0$; $i=3,4,5$. For a specified $k_{*}$, the form of $\beta^{+}, \gamma^{+}$, and $\delta^{+}$is then completely specified by two variables, $\sigma_{*}$ and $b_{1}^{+} / b_{2}^{+}$. These are adjusted so that when we integrate over the waveform, we satisfy the two conditions at large negative $y_{*}$, i.e., $b_{\overline{1}}=b_{\overline{2}}=0$. In our search, which was restricted to $\sigma_{*}>0$, this was only found to be possible for a finite range of $k$.

The results are shown in Figure 4. For comparison, in the numerical experiment shown in Figure 1 the initial amplitude $\Psi=1.8$, and the horizontal wave number $k_{*}=1.9$. In the early part of the experiment the growth rate $\sigma_{*}=0.17$. We see that the linear stability results are consistent with the finite amplitude results, and the assumption that the dominant instability is that which grows the fastest. Figure 4 also shows that for each $\Psi$, there is a maximum wave number for which any growth is possible. The decay of short wavelength noise between the first and second frames of Figure 1 is thereby understandable. 


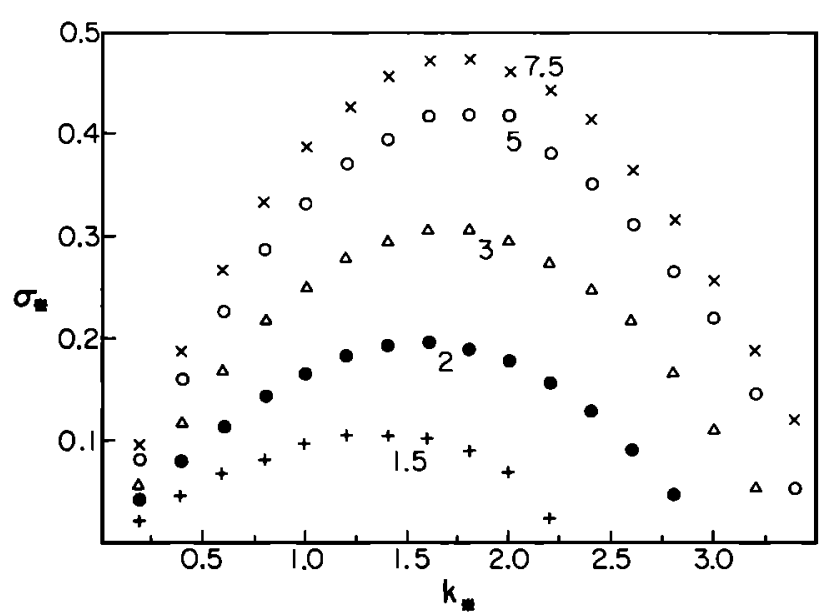

Fig. 4. Results of linear stability analysis for lateral perturbations of one-dimensional solitary waves. These results, from the analysis in section 4, pertain directly to the experiment shown in Figure 1. $\sigma$. is the growth rate of a harmonic lateral instability, in units of $c / 2\left(2 c \varphi_{\max }\right)^{1 / 2}$ (see text for discussion of the nondimensionalization), and $k_{*}$ is the horizontal wavenumber in units of $1 / 2\left(2 c \varphi_{\max }\right)^{1 / 2}$. The individual curves are labeled by $\Psi$, the wave amplitude relative to the background porosity. The finite amplitude results in Figure 1 correspond to $k_{*}=1.9, \sigma_{*}=0.17$, and $\Psi=1.8$, consistent with the peak of the corresponding curve here.

\section{Analysis of Two-Dimensional Solitary Waves}

In our earlier report on the one-dimensional system [Scott and Stevenson, 1984] we were able to show a close correspondence between numerical experiments and analytical theory. Despite the greater difficulty of analyzing the two-dimensional system, we are able to show that solitary waves of circular cross section exist with the form and phase speed implied by the numerical results.

We proceed by rewriting equations (6), (7a) and $(7 b)$ in the form

$$
\begin{gathered}
f_{t}-w_{z}+u_{x} \\
w=f^{n}\left[-1+(1-\alpha) f_{t z}+\alpha \nabla^{2} w\right] \\
u=f^{n}\left[(1-\alpha) f_{t x}+\alpha \nabla^{2} u\right]
\end{gathered}
$$

We seek a solution to equations (15), (16a), and (16b) in which the porosity takes the form of a propagating wave of circular cross section:

$$
\begin{gathered}
f(x, y, t)=\varphi(\xi) \\
\xi^{2} \equiv x^{2}+y^{2} \quad y \equiv z-c t
\end{gathered}
$$

It then follows that

$$
\begin{gathered}
f_{t z}=-\frac{c}{\xi} \varphi^{\prime}-\frac{c y^{2}}{\xi^{3}}\left(\xi \varphi^{\prime \prime}-\varphi^{\prime}\right) \\
f_{L x}=-\frac{c x y}{\xi^{3}}\left(\xi \varphi^{\prime \prime}-\varphi^{\prime}\right)
\end{gathered}
$$

where a prime denotes differentiation with respect to $\xi$. On comparison with equations $(16 a)$ and $(16 b)$ these suggest the following trial solutions for the velocity components:

$$
\begin{gathered}
w=\omega(\xi)+y^{2} \chi(\xi) \\
u=x y \chi(\xi)
\end{gathered}
$$

The unknown functions $\varphi(\xi), \omega(\xi)$, and $\chi(\xi)$ are found to satisfy the following three coupled, nonlinear ordinary differential equations:

$$
\begin{gathered}
-\frac{c}{\xi} \varphi^{\prime}=\frac{1}{\xi} \omega^{\prime}+3 \chi+\xi \chi^{\prime} \\
\omega=\varphi^{n}\left[-1-(1-\alpha) \frac{c}{\xi} \varphi^{\prime}+\alpha\left[2 \chi+\omega^{\prime \prime}+\frac{1}{\xi} \omega^{\prime}\right]\right] \\
\chi=\varphi^{n}\left[(1-\alpha) \frac{c}{\xi^{3}}\left(\varphi^{\prime}-\xi \varphi^{\prime \prime}\right)+\alpha\left[\chi^{\prime \prime}+\frac{5}{\xi} \chi^{\prime}\right]\right]
\end{gathered}
$$

Equation (20) is obtained by substitution of the trial solutions into (15). Substitution of $(18 a)$ and (19a) into (16a) gives an equation with terms involving only $\xi$, and terms that include $y^{2}$; separation of these two groups gives equations (21a) and (21b). This separation must be permissible if circular solutions, dependent only on $\xi$, exist. Substitution of $(18 b)$ and $(19 b)$ into $(16 b)$ duplicates equation $(21 b)$, demonstrating that the above procedure is consistent.

The circular solitary wave solution defined by the functions $\varphi, \omega$, and $\chi$ may not be unique. However, we can show that solutions with elliptical cross sections $\left(\xi^{2} \equiv x^{2}+A y^{2}, A \neq 1\right)$ do not exist, and we suggest that circular cross sections are preferred.

Equations (20), (21a), and (21b) were solved numerically by a shooting method, integrating from small $\xi$ (the peak of the wave) out to large $\xi$ (the background). The Taylor series expansions for $\varphi, \omega$, and $\chi$, at small $\xi$, were adjusted until a decaying solution was obtained as $\xi \rightarrow \infty$. The asymptotic forms of the functions at large $\xi$ are modified Bessel functions of the second kind. The solutions thus obtained agree with the finite difference solutions to within a few percent in porosity profile, the velocity components, and the phase velocity of the wave. The finite difference results also agree with the functional forms in equations $(19 a)$ and $(19 b)$.

\section{QUANTIFICATION}

We are confident that the qualitative aspects of our results are robust. For this reason, the speculative assignment of physical dimensions to these results is reserved for this separate section. The importance of magma ascent by porous flow, and particularly in magmons, is of course dependent on such assignments. (At this point we revert to use of the term "magmon" when referring to the solitary wave solutions). We present our preferred values in Table 1.

Figure $5 a-5 d$ shows the variation of magmon ascent velocities $c$ and heights $h$ with amplitude. The velocity plots were produced using the one-dimensional dispersion relations presented by Scott and Stevenson [1984]. Empirically, these are found to provide good estimates of the ascent velocities of magmons in two and three dimensions. The heights are the half heights of approximate solutions to the one-dimensional equations:

$$
h=\left[2 c f_{\max }\right]^{1 / 2}(m=0) \quad h=[2 c]^{1 / 2} \quad(m=1)
$$

The approximation is good in the large-amplitude limit and provides a lower bound for small amplitudes. The conclusion from these calculations is that magmon half heights of at least $1 \mathrm{~km}$, and magmon velocities of at least $1 \mathrm{~cm} \mathrm{yr}$, 
TABLE 1. Parameters, Definitions, and Values

\begin{tabular}{|c|c|c|c|c|}
\hline Parameter & $\begin{array}{c}\text { Symbol } \\
\text { (Definition) }\end{array}$ & \multicolumn{2}{|c|}{ Value } & Units \\
\hline $\begin{array}{l}\text { Porosity (liquid fraction) } \\
\text { Bulk viscosity } \\
\text { Shear viscosity } \\
\text { Liquid viscosity } \\
\text { Gravity } \\
\text { Density contrast } \\
\text { Grain radius }\end{array}$ & $\begin{array}{c}f \\
\zeta \\
\eta \\
\eta^{7} \\
g \\
\Delta \rho=\rho^{s}-\rho^{l} \\
a\end{array}$ & & & $\begin{array}{l}\text { none } \\
\mathrm{Pa} \mathrm{s} \\
\mathrm{Pa} \mathrm{s} \\
\mathrm{Pa} \mathrm{s} \\
\mathrm{m} \mathrm{s}^{-2} \\
\mathrm{~kg} \mathrm{~m}^{-3} \\
\mathrm{~m}\end{array}$ \\
\hline Permeability $^{a}$ & $\begin{array}{c}k-a^{2} f^{n} / b \\
n \\
b\end{array}$ & $\begin{array}{c}2 \\
10^{4}\end{array}$ & $\begin{array}{c}3 \\
10^{3}\end{array}$ & $\begin{array}{l}\mathrm{m}^{2} \\
\text { none } \\
\text { none }\end{array}$ \\
\hline Length scale ${ }^{b}$ & $L=a\left[(\zeta+4 / 3 \eta) / b \eta^{\prime}\right]^{1 / 2}$ & 10 & 30 & $\mathrm{~km}$ \\
\hline Time scale & $T=\frac{1}{g \Delta \rho a}\left[(\zeta+4 / 3 \eta) b \eta^{l}\right]^{1 / 2}$ & $3 \times 10^{4}$ & $10^{4}$ & $\mathrm{yr}$ \\
\hline Velocity scale ${ }^{b}$ & $L / T-g \Delta \rho a^{2} / b \eta^{\prime}$ & 30 & 300 & $\mathrm{~cm} \mathrm{yr}^{-1}$ \\
\hline $\begin{array}{l}\text { Compaction length } \\
\text { Compaction time } e^{c}\end{array}$ & $\begin{array}{c}\delta_{c}=L f^{(n-m) / 2} \\
\tau_{0}=T f^{1-(n+m) / 2}\end{array}$ & & & \\
\hline
\end{tabular}

This table shows various parameter choices, all of which influence the values of the length, time, and velocity scales used to non-dimensionalize the governing equations.

$a$ The choices of the parameters $n$ and $b$ in the permeability provide two fits to the data of Maaloe and Scheie [1982]. The change in the value of $b$ alters the length, time and velocity scales as shown.

$b$ We consider the numerical values given for the length and velocity scales to be lower bounds because of our conservative choices for the grain size and density contrast. Note that the velocity scale varies as the square of the grain size.

$c$ The expressions for the compaction length and time are provided for continuity with Scott and Stevenson [1984] and McKenzie [1984]. The exponent $m$ describes the functional dependence of the matrix viscosity on porosity (see Appendix B).

are to be expected. The volume of liquid within a magmon of this size, with an amplitude of about $10 \%$, is about 1 $\mathrm{km}^{3}$. The maximum value of the pressure difference between the liquid and solid matrix is a few bars.

\section{Discussion}

The focus of this discussion is an assessment of the likely importance, in the earth, of magma ascent in magmons. We must first discuss the incorporation of the actual melting into our modeling of magma ascent.

The actual process of melting is not included in the equations we have been modeling. We justify our continuing study of the system without the inclusion of melting as follows. Coupling the melting process into the system has two effects: (1) The addition of an equation for the conservation of energy, balancing the heat capacity and the latent meat of melting. This is coupled to the momentum equations by the heat supplied from viscous dissipation, but this is a small term (as is thermal conduction). (2) The addition of a source term in the continuity equation, representing the time derivative of porosity due to melting. This dominates the coupling between melting and magma migration.

This leads us to identify two end-member regimes, in which the nature of magma ascent by porous flow is very different. The first is that in which the flow process is dominated by the rate of supply of liquid by melting throughout the region of interest. As discussed below, this certainly applies beneath spreading centers. The second is that in which liquid is supplied to a stable partially molten region from below, and this supply dominates over melting within the region itself. This may apply in regions of the asthenosphere away from spreading centers, when supplied with liquid from below. In this case, magma is expected to ascend in magmons.

The transition between these two end-members may be quantified as follows. For a magmon to retain its identity, the background liquid fraction $f_{0}$ must change slowly relative to the rate of ascent of the magmon. Expressing as an inequality, we obtain

$$
\frac{1}{f_{0}} \frac{\partial f_{0}}{\partial t} \ll \frac{c}{h}
$$

The usefulness of this inequality has been demonstrated by experimentation on the one-dimensional system of equations, with melting included. If we attribute the melting $\partial f_{0} / \partial t$ to adiabatic ascent of the partially molten rock as a whole, at velocity $W$, we find

$$
W \ll 3 \mathrm{~cm} \mathrm{yr}^{-1}
$$

We have taken $n=3, m=0, f_{0}=1 \%, f_{\max }=10 \%$, and $0.3 \% / \mathrm{km}$ as the adiabatic rate of melting. We conclude that magmons can only survive in partially molten regions of the mantle that are not undergoing significant ascent. 
(a)

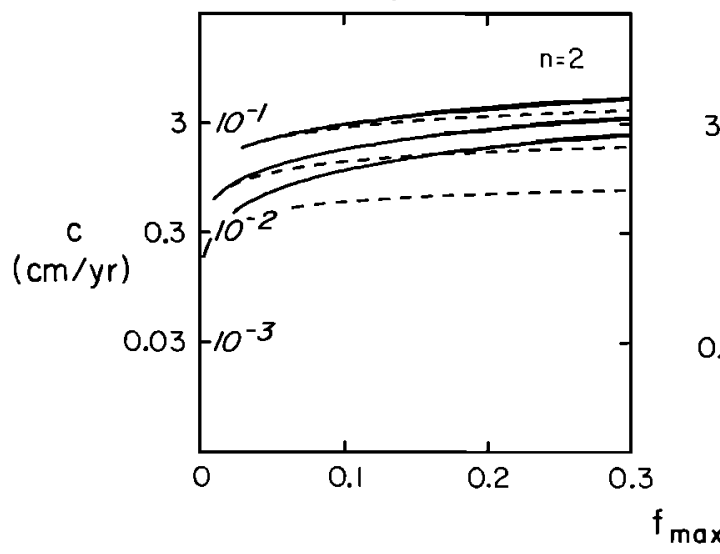

(c)

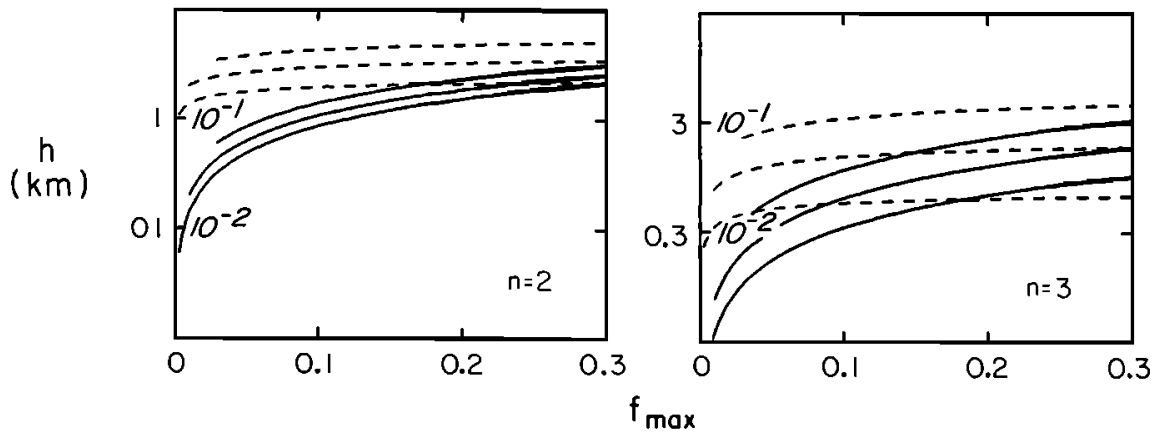

(b)

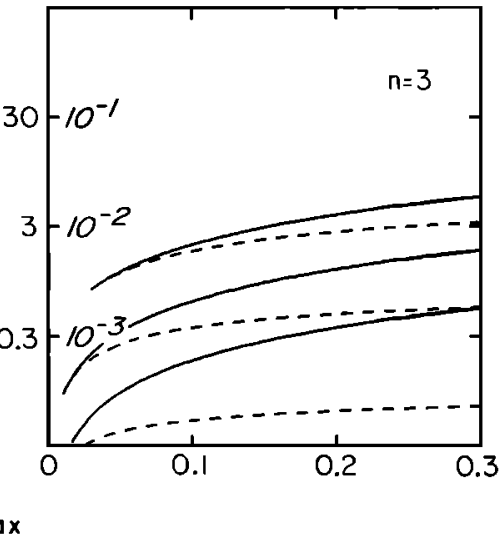

(d)

Fig. 5. Magmon heights and ascent velocities. These plots show the ascent velocity and approximate half height of magmons for a range of parameter choices. See Table 1 for definitions of the parameters. Figures $5 a$ and $5 b$ show the ascent velocity $c$ plotted against the maximum amplitude $f_{\max }$ of the waveform, on $\log$-linear axes. In Figure $5 a$, $n=2$ is used for the permeability; in Figure $5 b, n=3$ is used. For the solid lines, $m=0$ is used for the matrix viscosity; for dashed lines, $m-1$ is used. In each case, three values of $f_{0}$ are used; $f_{0}=3 \%, 1 \%$, and $0.3 \%$. The larger values of $f_{0}$ correspond to larger values of $c$. The vertical axes have two calibrations; the italic numerals within the axes are the non-dimensional values of $c$, and the roman numerals are dimensional values, in centimeters per year. Note that the dimensional calibration is different on Figures $5 a$ and $5 b$ because the velocity scale in Table 1 is different for $n=2$ and $n-3$. Figures $5 c$ and $5 d$ show the half heights of magmons, for the same range of parameters as Figures $5 a$ and $5 b$. Again, the dimensional calibration is different for $n-2$ and $n-3$. The experiments shown in Figures 1,2 , and 3 use $n=3, m=0$ and $f_{0}-1 \%$. These choices correspond to the central solid line in Figures $5 b$ and $5 d$. The values of $f_{m a x}$ in these experiments are in the region below $5 \%$.

The problem of melting due to steady, adiabatic decompression was originally addressed by Turcotte and Ahern [1978] and has been reviewed by Ribe [1985] in the light of recent work on porous flow and compaction. The conclusion is that in the scenario of a broad mantle upwelling beneath a spreading center, compaction is not important because the rate of magma escape adjusts to match the rate of magma production. In this setting, the thermal structure is dominated by the buffering effect of the phase change. Heat transfer by conduction is negligible within the melting regime, and heat transfer by movement of the magma is tempered because the magma is everywhere in intimate thermal contact with the matrix. To this we should add that due to the pervasive nature of the melting, magma ascent in magmons is unlikely to occur beneath ridges. To relate petrological and geochemical observables to processes in the mantle beneath ridges, the approaches of McKenzie [1985] and O'Hara [1985] are certainly correct.

If, in the light of the above, magmons exist anywhere, they should have some impact on geological observables. A simple suggestion is that the episodicity of the ascent pro- cess may translate into episodicity in the emplacement of the magma at or near the earth's surface. The episodic ascent could cause either temporal or spatial periodicity in emplacement. However, we do not propose that porous flow delivers magma all the way to the earth's surface. Therefore it is not clear that the associated periodicity could be detectable after the operation of high-level transport processes.

In the caption to Figure 3, we describe the exchange of liquid between large and small solitary waves during collisions. Solitary waves also exchange liquid with the background region as they ascend. Consider the velocities of the waveform and of the liquid at various points. In the background region, the liquid ascends uniformly at the Darcy flow velocity corresponding to $f_{0}$. The waveform ascends faster than the background (the relative velocity is the quantity $c^{*}$ used by Scott and Stevenson [1984]). The ascent velocity of the liquid is greatest at the peak of the wave but is still less than that of the waveform. Consequently, the waveform takes in new liquid from above and loses its original liquid from below. The rate at which this replacement 
proceeds depends on the amplitude of the wave; a larger wave will need to traverse more background liquid before it fills up. We note that flow of liquid through the matrix always follows streamlines, which means that mixing between the different liquids does not occur, even within the magmon.

This exchange has interesting implications for the chemical characteristics of magma delivered from a magmon. Suppose that a magmon ascends through a region where the liquid in the background is in equilibrium with the matrix. As the original liquid in the magmon is replaced with liquid from the background, this new liquid will remain in equilibrium with the matrix. This is because the equilibrium depends on concentrations; the effect of the passage of a magmon is to change only the volume fraction of liquid by collecting liquid from a vertically extensive region into one place. Conversely, the liquid originally in the magmon is left spread out in the trail of the magmon. This applies to both major and minor elements. The effect is that if the background liquid has a chemical signature corresponding to a small degree of partial melting, the magmon will deliver a large volume of this liquid in a short time. This process is akin to zone refining, which Harris [1957] has proposed to explain the origin of potassic basalts. We prefer to emphasize the more general conclusion that caution is needed in deducing source region sizes and time scales for magma escape, from petrological and geochemical observations.

We have discussed the conditions for survival of magmons, the possible effect on the periodicity of igneous emplacement, and the mechanism by which small degrees of partial melt may be collected and mobilized. In the light of these, we suggest that magma ascent in magmons may occur beneath oceanic volcanic centers or in the subcontinental mantle.

APPENDIX A:

Formulation of the Equations Governing Compaction

We think of the partially molten system as a spongelike crystalline matrix saturated with liquid. The porosity of the matrix phase and the volume fraction of liquid are therefore synonymous. Both the matrix material and the liquid are assumed to be fully connected. We use averaged values of the variables such as porosity, velocity, and stress. This averaging is performed on a scale much larger than the grain size but much smaller than the scale of compaction processes. We also assume that the Reynolds number is small for the flow of both phases, so that all inertial terms may be neglected.

First we define a local frame of reference for the twophase system. A suitable choice is the barycentric frame in which the velocities of the matrix $\mathbf{V}$, and liquid $\mathbf{v}$, are related by

$$
f v_{i}=-(1-f) V_{i}
$$

In this frame the volume fluxes of the liquid and matrix through any elemental surface are equal and opposite. The relationship between this local frame and a fixed global frame will be discussed later. The statement of continuity of the two phases, as the liquid fraction changes, is

$$
\frac{\partial f}{\partial t}=\frac{\partial}{\partial x_{l}}\left[(1-f) V_{l}\right]
$$

To describe the response of the matrix during compaction, we use the constitutive equation discussed in the text:

$$
\sigma_{i j}+p^{l} \delta_{i j}=\sigma_{i j}^{\prime}+\left(p^{l}-p^{s}\right) \delta_{l j}=(\zeta-2 / 3 \eta) e_{k k} \delta_{i j}+2 \eta \dot{e}_{i j}
$$

The stress deviator $\sigma_{i j}^{\prime}$ is trace free and the strain rate tensor $\dot{e}_{i j}$ is defined in terms of the velocity field in the matrix $\mathbf{V}$, in the usual way. The bulk viscosity $\zeta$ and the shear viscosity $\eta$ are defined by

$$
\begin{gathered}
p^{l}-p^{s}=\zeta \dot{e}_{k k} \\
\sigma_{i j}^{\prime}=2 \eta \dot{e}_{i j} \quad(i \neq j)
\end{gathered}
$$

Both $\zeta$ and $\eta$ may be functions of $f$. The constitutive relation is like that used for a compressible fluid, except that the liquid pressure $p^{l}$ appears as a reference for the pressure in the solid matrix $p^{s}$. Compaction should not occur when these are equal. McKenzie [1984] defines a single pressure for both phases, equal to the liquid pressure, and allows the stress deviator for the matrix to have a non-zero trace. The model presented in Appendix B illustrates our alternative approach.

We now seek to produce an equation for the conservation of mechanical energy during compaction. This does not include thermodynamic considerations such as temperature, heat capacity, and latent heat; the objective is to produce momentum equations describing the mechanical process of compaction. Consider an element of the two-phase mixture, large compared to the grain size but small compared to the scale of variations in the mean velocity field. The rate of work done on this element is described by surface integrals over the solid and liquid parts of the surface of the element:

$$
\begin{aligned}
\text { Power } & =\int_{s} \sigma_{i j} V_{l} d S_{j}-\int_{l} p^{l} \delta_{l \jmath} v_{l} d S_{j} \\
& =\int\left[(1-F) \sigma_{l \jmath} V_{i}-F p^{l} \delta_{l j} v_{l}\right] d S_{J}
\end{aligned}
$$

The final integral is over the entire surface of the element. Here $F$ is the "surface porosity"; to proceed we must assume that this is equal to the volume porosity $f$ of the same element. Using (AI) to eliminate the liquid velocity, we obtain

$$
\text { Power }=-\int(1-f)\left[\sigma_{i j}+p^{l} \delta_{t \jmath}\right] V_{\imath} d S_{J}
$$

By the divergence theorem:

$$
\text { Power per unit volume }=\frac{\partial}{\partial x_{j}}\left[(1-f)\left(\sigma_{t j}+p^{l} \delta_{i j}\right) V_{t}\right]
$$

This step provides an illustration of the assumptions related to averaging. The local flow associated with compaction is expected to vary on the scale of the grain size. We are using the divergence theorem on a volume element that is small compared to variations in the mean velocity field but large compared to these local fluctuations.

It will prove to be convenient to expand (A7) as follows:

Power per unit volume $=(1-f) \frac{\partial V_{i}}{\partial x_{j}}\left[\left(\sigma_{i j}+p^{\prime} \delta_{i j}\right]\right.$ 


$$
+V_{i} \frac{\partial}{\partial x_{j}}\left[(1-f)\left(\sigma_{i j}+p^{l} \delta_{i j}\right)\right]
$$

The rate of viscous dissipation in the deforming matrix is given by

$$
(1-f)\left[\sigma_{i j}^{\prime} \dot{e}_{i j}+\left(p^{l}-p^{s}\right) \dot{e}_{k k} \delta_{i j}\right]=(1-f) \dot{e}_{i j}\left[\sigma_{i j}+p^{l} \delta_{i j}\right]
$$

where $\dot{e}_{i j}$ is the strain rate. On the left-hand side, we use our understanding of the matrix rheology to divide to viscous dissipation in the matrix into two parts, corresponding to shear and compaction. An underlying assumption is that the cross terms between shear stress and compaction strain, and vice versa, sum to zero when locally averaged. Note that since the stress tensor is symmetric, we will later be able to cancel the right-hand side of (A9) with the first term on the right-hand side of (A8).

The rate of viscous dissipation in the liquid must scale as the square of the velocity difference between the liquid and solid matrix:

$$
\text { Rate of dissipation in the liquid }-(1-f) \frac{\eta^{\prime}}{k} V_{i} V_{i}
$$

We choose this form so that our final equation will be consistent with the form of Darcy's law for buoyancy-driven porous flow, without compaction, given by Turcotte and Ahern [1978]:

$$
f\left(v_{i}-V_{\imath}\right)=-V_{l}=\frac{k}{\eta_{l}} g \Delta \rho \delta_{i z}
$$

This defines the permeability $k$. D. P. McKenzie (personal communication, 1985) has noted that in the original experimental form of Darcy's law, $\partial p^{l} / \partial x_{i}$ replaces $g \Delta \rho \delta_{i z}$ in (A11). This leads to an additional factor of (1-f) multiplying $k$ when Darcy's law is presented in the form of (A11). This redefinition is quantitatively unimportant when $f \ll<$, because of the strong variation of $k$ with $f$ given below in (A12).

Recalling the discussion of permeability in the text, we use a simple functional dependence of $k$ on $f$ :

$$
k=\frac{a^{2} f^{n}}{b}
$$

where $a$ is the grain size and $n$ and $b$ are parameters. The variation of $k$ with $f$ is a source of nonlinearity in the final equations, because exponent $n$ has a value of at least 2 .

The system is driven by the release of gravitational energy. The rate of release is

$$
-\delta_{i z} g\left[f \rho^{l} v_{i}+(1-f) \rho^{s} V_{i}\right]=-g \Delta \rho(1-f) V_{i} \delta_{i z}
$$

The algebraic form of Darcy's law (A11) can be recovered using (A10) and (A13), ignoring the effects of matrix deformation.

The equation for the conservation of mechanical energy is obtained using the expressions for the dissipation in the two phases, (A9) and (A10); the rate of work (A8); and the rate of release of gravitational energy (A13):

$$
(1-f) \frac{\eta^{l}}{k} V_{i} V_{\imath}=-g \Delta \rho(1-f) V_{i} \delta_{i z}
$$

$$
+V_{i} \frac{\partial}{\partial x_{j}}\left[(1-f)\left(\sigma_{i j}+p^{l} \delta_{i j}\right)\right]
$$

We can extract the equations describing conservation of momentum from this statement of conservation of mechanical energy by bringing out the common factor of $V_{i}$ :

$$
V_{i}=\frac{k}{\eta^{l}}\left[-g \Delta \rho \delta_{i z}+\frac{1}{(1-f)} \frac{\partial}{\partial x_{j}}\left[(1-f)\left(\sigma_{i j}+p^{l} \delta_{i j}\right)\right]\right]
$$

or

$$
\begin{aligned}
V_{i}=\frac{k}{\eta^{l}}\left[-g \Delta \rho \delta_{i z}+\frac{\partial}{\partial x_{j}}\right. & \left(\sigma_{i j}+p^{l} \delta_{i j}\right) \\
& \left.-\frac{1}{(1-f)} \frac{\partial f}{\partial x_{j}}\left(\sigma_{i j}+p^{l} \delta_{i j}\right)\right]
\end{aligned}
$$

This equation may be obtained when those given by McKenzie [1984, equations A20 and A21] are placed in the barycentric frame, except that our $k$ is equivalent to $k(1-f)$ in his version. This is an inconsequential difference that can be absorbed into the definition of $k$. The expression in the square brackets is the pressure gradient that is available to drive relative flow of the liquid and matrix. The three terms are buoyancy, matrix compaction, and force between the two phases due to gradients in $f$. We note that the latter term is $O(f)$ relative to the other two; for this reason we omitted it from the momentum equation in our earlier report [Scott and Stevenson, 1984]. As mentioned in the text, additional terms of $O(f)$ might arise through changes in the way the equations are formulated. Here we have obtained (A15) by considering the conservation of mechanical energy; it may also be obtained by balancing the surface and body forces on an element of the two-phase medium, with the same assumptions of creeping flow and averaging.

The closed system of governing equations in the variables $f$ and $\mathbf{V}$ consists of (A2), (A3), (A12), and (A15). These may be non-dimensionalized using the dimensional scales $L$ and $T$ in Table 1 . These equations were derived in a specific, local frame of reference. In general, there may be relative motion between the barycentric frames defined in different localities. The inclusion of the dynamics of these motions leads to the complete set of governing equations given by McKenzie [1984]. For the calculations presented in this paper, we have assumed that these motions are negligible and have solved the local governing equations in a fixed global frame. This approximation is discussed further in section 3 of the text.

We present the momentum equations for the constant viscosity case, with the stresses expressed explicitly in terms of velocity gradients. For two-dimensional Cartesian coordinates, the components of matrix velocity in the vertical $(z)$ and horizontal $(x)$ directions are $w$ and $u$ respectively. Subscripts indicate partial differentiation with respect to the spatial coordinates:

$$
\begin{aligned}
& w=\frac{k}{\eta^{l}}\left[-g \Delta \rho+(\zeta+4 / 3 \eta) w_{z z}+\eta w_{x x}+(\zeta+1 / 3 \eta) u_{x z}\right. \\
& \left.-\frac{f_{z}}{(1-f)}\left[(\zeta+4 / 3 \eta) w_{z}+(\zeta-2 / 3 \eta) u_{x}\right]-\frac{f_{x}}{(1-f)} \eta\left(w_{x}+u_{z}\right)\right] \\
& u=\frac{k}{\eta^{l}}\left[(\zeta+4 / 3 \eta) u_{x x}+\eta u_{z z}+(\zeta+1 / 3 \eta) w_{x z}\right.
\end{aligned}
$$




$$
\left.-\frac{f_{x}}{(1-f)}\left[(\zeta+4 / 3 \eta) u_{x}+(\zeta-2 / 3 \eta) w_{z}\right]-\frac{f_{z}}{(1-f)} \eta\left(u_{z}+w_{x}\right)\right]
$$

For cylindrical polar coordinates, with no azimuthal variation, the components of matrix velocity in the vertical $(z)$ and radial $(r)$ directions are $w$ and $v$ :

$$
\begin{aligned}
& w=\frac{k}{\eta^{l}}\left[-g \Delta \rho+(\zeta+4 / 3 \eta) w_{z z}+\eta\left(w_{r}+\frac{w_{r}}{r}\right)\right. \\
& +(\zeta+1 / 3 \eta)\left(v_{r z}+\frac{v_{z}}{r}\right)-\frac{f_{r}}{(1-f)} \eta\left(w_{r}+v_{z}\right) \\
& \left.-\frac{f_{z}}{(1-f)}\left[(\zeta+4 / 3 \eta) w_{z}+(\zeta-2 / 3 \eta)\left(v_{r}+\frac{v}{r}\right)\right]\right] \\
& v=\frac{k}{\eta^{l}}\left[(\zeta+4 / 3 \eta)\left(v_{r r}+\frac{v_{r}}{r}-\frac{v}{r^{2}}\right)+\eta v_{z z}+(\zeta+1 / 3 \eta) w_{r z}\right. \\
& \left.-\frac{f_{r}}{(1-f)}\left[(\zeta+4 / 3 \eta) v_{r}+(\zeta-2 / 3 \eta)\left(w_{z}+\frac{v}{r}\right)\right]-\frac{f_{z}}{(1-f)} \eta\left(v_{z}+w_{r}\right)\right]
\end{aligned}
$$

(A17b)

\section{APPENDIX B}

\section{Model of One-Dimensional Compaction}

Consider an infinite, vertical column of solid, with a hexagonal cross section of area $\pi R_{0}^{2}$. At the center of the column is a vertical tubule of circular cross-section and radius $R_{i} \ll<R_{0}$. We fill all space with similar columns, such that any horizontal plane is tessellated by hexagons, and the tubules form a triangular lattice. Each tubule of this model porous matrix is filled with liquid. The solid and liquid phases are separately incompressible and of very similar density.

We wish to solve for the flow field in the solid during compaction, i.e., as $R_{l}$ changes. Since $R_{l} \ll<R_{o}$, it is a good approximation to replace the hexagonal boundary of each column with a circular boundary of radius $R_{o}$. This permits us to use cylindrical coordinates $(z, r)$ centered on the axis of the tubule. The corresponding components of flow in the solid are $w$ and $v$. The complete set of equations governing flow in the solid is

$$
\begin{gathered}
\frac{1}{r} \frac{\partial}{\partial r}(r v)+\frac{\partial w}{\partial z}=0 \\
\frac{\partial}{\partial r}\left[\frac{1}{r} \frac{\partial}{\partial r}(r v)\right)+\frac{\partial^{2} v}{\partial z^{2}}=\frac{1}{\eta^{s}} \frac{\partial p^{s}}{\partial r} \\
\frac{1}{r} \frac{\partial}{\partial r}\left[r \frac{\partial w}{\partial r}\right)+\frac{\partial^{2} w}{\partial z^{2}}=\frac{1}{\eta^{s}}\left(\frac{\partial p^{s}}{\partial z}+\rho^{s} g\right)
\end{gathered}
$$

where $p^{s}$ and $p^{l}$ are the pressures of the solid and liquid, $\rho^{s}$ and $\eta^{s}$ are the density and viscosity of the solid, and $g$ is the acceleration due to gravity. Equation (B1) expresses continuity of the deforming solid. Equations (B2a) and (B2b) are the components of the Navier-Stokes equation.

The boundary conditions are

$$
\begin{gathered}
v\left(R_{o}\right)=0 \\
{\left[-p^{s}+2 \eta^{s} \frac{\partial v}{\partial r}\right]_{R}=-p^{l}}
\end{gathered}
$$

$$
\left[\frac{\partial v}{\partial z}+\frac{\partial w}{\partial r}\right]_{R}=0
$$

The outer boundary condition (B3) is consistent with the incompressibility of both phases and horizontal uniformity. Equation (B4) is the balance of normal stress at the tubule wall, assuming that the wall is vertical. Equation (B5) is the balance of tangential stress at the tubule wall, assuming that $\eta^{\prime}<<\eta^{s}$. Notice that we do not need to impose conditions on the stress at the outer boundary.

By inspection, these equations are satisfied to high accuracy by "plug flow" in which $w$ and $p^{s}$ are independent of $r$ and

$$
\mathrm{v}=\frac{\left(R_{o}^{2}-r^{2}\right)}{2 r} \frac{d w}{d z}
$$

The justification for all the approximations leading to (B6) is that $R_{o} \ll L$, where $L$ is the macroscopic length scale over which compaction is taking place. This inequality enables us to neglect deviations of the tubule wall from the vertical. Since $L / R_{o}$ varies as $\left(\eta^{s} / \eta^{l}\right)^{1 / 2}$ this inequality is satisfied when $\eta^{l}<<\eta^{s}$. It follows from equation (B4) that

$$
p^{l}-p^{s}=\eta^{*} \frac{d w}{d z}
$$

which shows explicitly how compaction and the pressure difference are related. This equation defines a ' compaction viscosity $\eta^{*}$ for this simple model:

$$
\eta^{*} \equiv\left[\frac{1}{f}+1\right\} \eta^{s}
$$

Comparison with equations derived in Appendix A shows that $\eta^{*}$ corresponds to the composite quantity $\zeta+4 / 3 \eta$. To complete the specification of the simple model, we include the Poiseuille flow of the liquid:

$$
w^{l}(r)-w=-\frac{\left(R_{i}^{2}-r^{2}\right)}{4 \eta_{l}}\left[\frac{d p^{l}}{d z}+\rho^{l} g\right]
$$

and impose conservation of volume:

$$
\int_{0}^{R_{1}} w^{l} r d r+\int_{R_{1}}^{R_{0}} w r d r=0
$$

The superscripts 1 refer to quantities in the liquid. This leads, finally, to a modified Darcy's law in the form

$$
\left.w=\frac{f^{2} R_{o}^{2}}{8 \eta^{l}}\left[-g \Delta \rho+\frac{d}{d z} \mid \eta^{*} \frac{d w}{d z}\right]+\frac{f}{(1+f)} \eta^{*} \frac{d^{2} w}{d z^{2}}\right]
$$

where $f \equiv R_{l}^{2} / R_{o}^{2}$ is the liquid fraction and $\Delta \rho-\rho^{s}-\rho^{l}$.

A realistic model of the compaction process should include a complex tubule geometry and compaction occurring by grain-boundary transport processes as well as the continuum deformation of the solid in this simple model. This simple model illustrates three features that realistic models should share:

1. The pressure difference between the solid and liquid drives the compaction. This is true even if the compaction 
mechanism involves grain boundary transport or pressure solution.

2. Terms of order $f$ relative to the main terms (e.g., the third term on the right-hand side of equation (B11)) may exist but cannot be computed with confidence. Different assumptions in the above model are found to lead to different terms of this order. Thus different theoretical approaches are only likely to agree in the limit $f \rightarrow 0$.

3. The value of $\eta^{*}$ (and by inference $\zeta$ and $\eta$ ) may vary strongly with the porosity $f$, when $f<<1$. Scott and Stevenson [1984] proposed the functional form $\eta^{*}=\eta_{a}^{*} f^{-m}$, with the exponent $m$ varying between 0 and 1 .

The physically appealing but simplified nature of this model guides our formulation of a more complete theory presented in Appendix A. It is our belief, however, that no "exact" formulation is possible. For complex tubule geometries, unverifiable assumptions must be made in connecting the microscopic description (on the scale of the grain size $R_{o}$ ) to the macroscopic description (on the scale of compaction $L$ ).

\section{APPENDIX C:}

\section{Notes on the Numerical Method}

For this discussion, we use nondimensionalized versions of equations (A2), (A16a), and (A16b) from Appendix A, with the $O(f)$ terms omitted and with $\zeta=\eta$.

$$
\begin{gathered}
f_{t}-w_{z}+u_{x} \\
w-f^{n}\left[-1+w_{z z}+3 / 7 w_{x x}+4 / 7 u_{x z}\right] \\
u=f^{n}\left[u_{x x}+3 / 7 u_{z z}+4 / 7 w_{x z}\right]
\end{gathered}
$$

The first part of the algorithm solves equations ( $\mathrm{C} 2 a)$ and $(\mathrm{C} 2 b)$ for the velocity field, given the porosity distribution. We solve (C2a) for the vertical velocity and $(\mathrm{C} 2 b)$ for the lateral velocity, using the alternating direction implicit finite-difference method, with second-order estimates of the derivatives (see, for example, Press et al. [1986]). In this method, each equation is solved in each direction; the most recent values of the vertical and lateral velocity are used to estimate terms that are not included in the solution. For example, in solving ( $\mathrm{C} 2 a)$ in the z-direction, we solve for $w$ and $w_{z z}$, using estimates for the remaining terms. The solution consists of the inversion of a tridiagonal matrix of the values of the velocity field.

Boundary conditions on the velocity field are applied during this calculation. The models shown in this paper are concerned with the behavior of solitary waves within a region of uniform porosity, so no matrix deformation occurs near the boundaries. We have found that zero-slope boundary conditions at the external boundaries, on both components of the velocity, are suitable.

The second part of the algorithm is the advancement of $f$ in time. Given some distribution of melt at time $t$, we wish to find the distribution at time $t+\delta t$. The time derivative of porosity is given explicitly by equation (C1), in terms of the velocity field in the matrix and the porosity distribution. This is solved using explicit finite differences. Since the dominant terms in equation ( $\mathrm{Cl}$ ) are first derivatives of the velocity field, we define the velocity on a grid symmetrically offset from that of the porosity. To center the time deriva- tive at time $t+1 / 2 \delta t$, the quantities in equation (C1) must be estimated at that time. We use an iterative Lax-Wendroff method (see Press et al. [1986]). The centering is achieved by estimating of the porosity distribution at $t+1 / 2 \delta t$, and reevaluating the velocity field. This procedure is iterated until subsequent estimates of the centered time derivative converge. We have chosen to gauge this convergence by tracing the relative convergence of the largest value of the time derivative within the grid. Convergence to within 1 part in $10^{3}$ usually requires $2-4$ iterations.

The grid size was chosen after studying the exact analytic solution for the one-dimensional, $n=3$, constant viscosity case, and performing some experiments on the onedimensional system of equations. The minimum grid size that can be used is controlled by the sharpness of the transition between the uniform background region and the main body of the waveform. The grid heights used in the experiments shown here are: Figure 2, 60 points; Figure 3, 120 points; Figure 4, 100 points. In both the Cartesian and cylindrical cases the lateral and vertical grid spacings are equal. The time step is then chosen with reference to the maximum phase velocity expected to arise during the experiment. The calculations shown in this paper each took roughly 3 hours on a VAX 11/780.

All the calculations presented here were performed using a scrolling grid, in order to keep the solitary waves away from the top and bottom edges of the grid while minimizing computational time. The equations were, however, always solved in the barycentric frame. The scrolling is accomplished by adding a new row of grid points at the top of the grid between time steps and removing a row from the bottom. The new points all have $f=f_{0}$, the background porosity. This procedure is justified because we always ensure that nonuniform regions of porosity do not approach the edges of the grid.

The accuracy of the calculations have been assessed by repeating certain examples with different grid sizes. First, we have studied the effects of these changes on the solitary waves used in the initial state of Figure 4. We note the following:

1. Solitary waves that propagate for many wavelengths with changes in the waveform of less than 1 part in $10^{4}$ are always produced after some transient adjustment.

2. Halving the time step (from the maximum permitted by the velocity of the wave) changes the wave amplitude by less than $0.1 \%$.

3. Halving the grid size (by interpolating) and the time step changes the wave amplitude by less than $0.5 \%$.

The measured phase velocity also changes slightly. We expect some changes, corresponding to the changes in wave amplitude; the observed changes are comparable to those predicted from the dispersion relation for one-dimensional solitary waves.

Second, we have repeated the calculation shown in Figure $4 a$, with a halved grid size and time step. The phase shift of the larger wave that arises through the collision with the smaller wave changes by less than $2 \%$. Errors in the phase shift would accumulate through the several hundred time steps performed, so we consider this to be a searching test.

We also note that calculations have been performed using the complete form of equations (A2), (A16a), and (A16b), and using simplified forms with the $O(f)$ terms omitted. The latter were used for direct comparison with the analysis 
presented in sections 4 and 5 of the text. However, the differences that arise when $O(f)$ terms are included are small. In particular, inclusion of these terms has no effect on the existence or stability of solitary wave solutions.

Acknowledgments. We are grateful to numerous colleagues for comments and suggestions, in particular $R$. Clayton, B. Hager, $O$. Navon, M. O'Hara, E. Stolper, and R. Svendsen. Helpful reviews were provided by D. McKenzie and S. Jurewicz. This work was supported by National Science Foundation grant EAR-8418353 and NASA grant NSG-7610. Contribution 4201, Division of Geological and Planetary Science, California Institute of Technology, Pasadena.

\section{REFERENCES}

Arzi, A. A., Critical phenomena in the rheology of partially molten rocks, Tectonophysics, 44, 173-184, 1978.

Auer, F., H. Beckmeimer, and G. Oehlschlegel, Steady state creep of fine grain granite at partial melting, $J$. Geophys., 49, 89-92, 1981.

Carman, P. C., Fluid flow through granular beds, Trans. Inst. Chem. Eng., 15, 150-161, 1937.

Cooper, R. F., and D. L. Kohlstedt, Solution-precipitation enhanced diffusional creep of partially molten olivine-basalt aggregates during hot-pressing, Tectonophysics, 107, 207-233, 1984.

Fowler, A. C., On the transport of moisture in polythermal glaciers, Geophys. Astrophys. Fluid Dyn., 28, 99-140, 1984.

Harris, P. G., Zone refining and the origin of potassic basalts, Geochim. Cosmochim. Acta, 12, 195-208, 1957.

Maaløe, S., and A. Scheie, The permeability-controlled accumulation of primary magma, Contrib. Mineral. Petrol., 81, 350-357, 1982.

McKenzie, D. P., The generation and compaction of partial melts, J. Petrol., 25, 713-765, 1984.

McKenzie, D. P., ${ }^{230} \mathrm{Th}-{ }^{238} \mathrm{U}$ disequilibrium and the melting process beneath ridge axes, Earth Planet. Sci. Lett., 72, 149-157, 1985.

O'Hara, M., Importance of the 'shape' of the melting regime during partial melting of the mantle, Nature, 314, 58-62, 1985.

Press, W. H., B. P. Flannery, S. A. Teukolsky, and W. T. Vetterling, Numerical Recipes, 818pp, Cambridge University Press, 1986.
Ribe, N., The generation and compaction of partial melts in the earth's mantle, Earth Planet. Sci. Lett., 73, 361-376, 1985.

Richter, F. M., and D. P. McKenzie, Dynamical models for melt segregation from a deformable matrix, J. Geol., 92, 729-740, 1984.

Scott, D. R., and D. J. Stevenson, Magma solitons, Geophys. Res. Lett., 11, 1161-1164, 1984.

Shaw, H. R., The fracture mechanisms of magma transport from the mantle to the surface, in Physics of Magmatic Processes, edited by R. B. Hargraves, pp. 201-264, Princeton University Press, Princeton, N.J., 1980.

Spence, D. A. and D. L. Turcotte, Magma driven propagation of cracks, J. Geophys. Res., 90, 575-580, 1985.

Spera, F. J., Carbon dioxide in petrogenesis, III, Role of volatiles in the ascent of alkaline magmas with special reference to xenolith bearing mafic lavas, Contrib. Mineral. Petrol., 88, 217-232, 1984.

Stevenson, D. J., Magmatic transport by the migration of fluidfilled cracks, Eos Trans. $A G U, 64,848,1983$.

Toramaru, A., and N. Fujii, Connectivity of the melt phase in partially molten peridotite, J. Geophys. Res., this issue.

Turcotte, D. L., and J. L. Ahern, A porous flow model for magma migration in the asthenosphere, J. Geophys. Res., 83, 767-772, 1978.

Vaughan, P. J., D. J. Kohlstedt, and H. S. Waff, Distribution of the glass phase in hot-pressed, olivine-basalt aggregates: An electron microscopy study, Contrib. Mineral. Petrol., 81, 253-261, 1982.

Waff, H. S., Effects of the gravitational field on liquid distribution in partial melts within the upper mantle, J. Geophys. Res., 85, 1815-1825, 1980.

Waff, H. S., and J. R. Bulau, Equilibrium fluid distribution in an ultramafic partial melt under hydrostatic stress conditions, $J$. Geophys. Res., 84, 6109-6114, 1979.

Weertman, J., Theory of fluid-filled crevasses in glaciers applied to vertical magma transport beneath ridges, J. Geophys. Res., 76, $1171-1183,1971$.

D. R. Scott, Seismological Laboratory, 252-21, California Institute of Technology, Pasadena, CA 91125.

D. J. Stevenson, Division of Geological and Planetary Sciences, 170-25, California Institute of Technology, Pasadena, CA 91125.

(Received April 2, 1985; revised December 20, 1985; accepted January 3, 1986.) 